\title{
Rare Outcome of Lecompte's Maneuver: SVC Compression due to Retro Aortic Innominate Vein
}

Rajat Kalra*, Raja Joshi, Reena K Joshi and Neeraj Aggarwal

Department of Pediatric Cardiac Sciences, Sir Ganga Ram Hospital, New Delhi, India

\begin{abstract}
The impact of Lecompte's maneuver on superior vena cava (SVC) has never been mentioned before. We think that in certain anatomical subsets, Lecompte's maneuver can result in superior vena cava narrowing. We present a case report of patient with Tetralogy of Fallot with absent pulmonary valve with retro-aortic innominate vein who developed SVC thrombosis after Lecompte's maneuver.
\end{abstract}

Keywords: Lecompte's maneuver; SVC narrowing; congenital heart disease; Tetralogy of fallot with absent pulmonary valve

\section{Introduction}

Lecompte's maneuver is widely being used these days in variety of complex congenital cardiac repairs [1]. However, its implication on superior venacaval (SVC) dynamics has never been considered. Our intent is to discuss specific situations where Lecompte's maneuver can increase the risk of SVC thrombosis, so that appropriate preventive measures can be taken.

\section{Case Report}

A 5-week-old female was intubated at a peripheral medical center, in view of respiratory distress and shifted to our hospital for further management. On physical examination, patient was acyanotic with insignificant general examination. On cardiovascular examination, S1 was normal and P2 was absent. There was grade II crescendo decreasendo murmur in pulmonary area. Echocardiographic examination revealed Tetralogy of fallot with absent pulmonary valve. CT pulmonary angiography revealed aneurysmal dilatation of the mid RPA resulting in significant compression of the right bronchus. Note was also made of a retro-aortic innominate vein. Plan was made for intracardiac repair and Lecompte's maneuver to relieve right bronchial compression. Consent was taken for surgery. On induction Right femoral arterial and venous access was obtained. After instituting cardiopulmonary bypass and achieving good diastolic arrest, trans RA Dacron patch closure of VSD was done. Lecompte's maneuver was done after dividing aorta, bringing RPA anterior and inferior with respect to right bronchus. RVOT was reconstructed using pericardium. Patient was weaned off cardiopulmonary bypass and shifted to ICU in stable condition. Patient was kept intubated and mechanically ventilated postoperatively. Patient was extubated on fourth post operative day. Patient developed chest wall edema along with upper body swelling $10^{\text {th }}$ postoperative day. Patient also developed stridor and worsening respiratory distress over next $12 \mathrm{~h}$. Patient was electively ventilated due to evolving respiratory failure. Vocal cord appeared edematous at time of intubation, requiring a smaller size ET tube. Echocardiography revealed normal biventricular function with SVC thrombosis. Bronchoscopy showed normal right bronchus and chest $\mathrm{X}$-ray showed bilateral pleural effusion. Therefore, it was concluded that SVC occlusion along with vocal cord edema and pleural effusion lead to respiratory failure. Heparin infusion was started and plan was made to continue supportive treatment till SVC recannalize. However, patient developed nosocomial infection due to Kleibsella pneumoniae. Chest $\mathrm{X}$-ray revealed $\mathrm{B} / \mathrm{L}$ infiltrates $14^{\text {th }}$ postoperative day. Appropriate antibiotics were administered; however, patient developed multiorgan dysfunction and died on post-operative day 22, despite supportive treatment.

\section{Discussion}

Lecompte's maneuver is often used to bring pulmonary artery complex main pulmonary artery (MPA), intra-pericardial branch pulmonary arteries $\}$ anterior to aorta, in order to ease right ventricular outflow tract reconstruction (Figure 1). It is widely used in arterial switch operation. However, off late its indications have expanded [1]. It is increasingly being used in patients of tetralogy of fallots with absent pulmonary valve with airway obstruction [1]. The anterior translocation of MPA and antero-inferior realignment of branch pulmonary arteries with respect to bronchus, helps in relieving airway obstruction in these patients following Lecompte's maneuver. The impact of displacement of great vessels on SVC-innominate complex has hardly been ever addressed. The anterior and inferior displacement
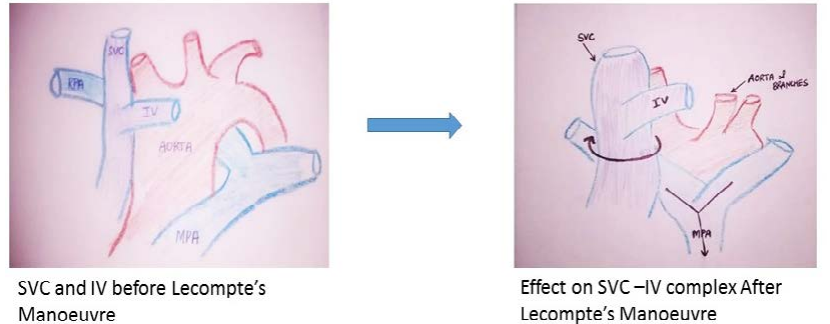

Lecompte's Manoeuvre

Figure 1: Leptome's manoeuvre result in anterior and inferior movement of MPA and branch pulmonary arteries along with lateral rotation of SVCinnominate vein complex.

RPA: Right Pulmonary Artery; IV: Innominate Vein; MPA; Main Pulmonary Artery.

*Corresponding author: Dr. Rajat Kalra, Department of Pediatric Cardiac Sciences, Sir Ganga Ram Hospital, New Delhi, India, Tel: 9560852200; E-mail: rajat_doc_ucms@yahoo.co.in

Received April 28, 2017; Accepted May 26, 2017; Published May 29, 2017

Citation: Kalra R, Joshi R, Joshi RK, Aggarwal N (2017) Rare Outcome of Lecompte's Maneuver: SVC Compression due to Retro Aortic Innominate Vein. J Vasc Med Surg 5: 314. doi: 10.4172/2329-6925.1000314

Copyright: ( 2017 Kalra R, et al. This is an open-access article distributed under the terms of the Creative Commons Attribution License, which permits unrestricted use, distribution, and reproduction in any medium, provided the original author and source are credited. 

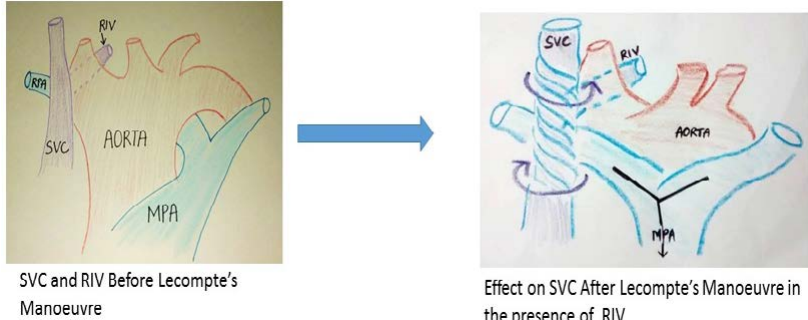

Effect on SVC After Lecompte's Manoeuvre in the presence of RIV

Figure 2: Retro aortic innominate vein limits the lateral rotation of svc due to the Leptome's manoeuvre. This results in twisting and narrowing of svc as MPA and branch pulmonary arteries move anterior and inferiorly on performing Leptome's manoeuvre.

RPA: Right Pulmonary Artery; RIV: Retro Aortic Innominate Vein; SVC: Superior Vena Cava; MPA; Main Pulmonary Artery.

of right pulmonary artery often leads to lateral displacement of SVCAzygous complex and anterior displacement of innominate vein (Figure 1). Good lateral mobilization and expansion of SVC is possible when innominate vein is in same plane as SVC. Therefore, the SVC drainage is not affected. However, we believe, that in certain subset of patients Lecompte's maneuver can lead to significantly increase risk of SVC obstruction and ensuing risk for thrombosis. First subsets are the patients with retro-aortic innominate vein, like the one we experienced in our patient. The lateral displacement of SVC is significantly impaired as anterior displacement of innominate vein is limited by the aorta. This leads to narrowing of SVC lumen (Figure 2). The compression of SVC by anteriorly displaced dilated right pulmonary artery further compromises the SVC lumen. The stasis due to significant reduction in SVC lumen size and endothelial injury as result of SVC cannulation can lead to propensity for thrombus formation in SVC. This possibly led to occurrence of SVC thrombosis is our patient.
Going by the above rationale, we think that similar SVC flow issue probably may occur in patients with IVC interruption and large azygous SVC connection. The large azygous may limit lateral mobilization of SVC and possibly lead to SVC narrowing and propensity for thrombus formation. However, we have not come across the second entity yet. Keeping this in mind, while performing Lecompte's maneuver with relatively immobile SVC situation, may help prevent serious postoperative problems due to SVC thrombosis.

We strongly believe in our hypothesis, as known causes of SVC thrombosis like presence of central line, traumatic cannulation of SVC were less likely in our patient. Central venous access was obtained in right femoral vein and not in SVC at the start of surgery. SVC was smoothly cannulated with $12 \mathrm{~F}$ angled venous cannula before institution of cardiopulmonary bypass. Moreover, we have performed 130 Lecompte's maneuver for various indications, but this is our first case where we encountered retro-aortic innominate vein as well as SVC thrombosis.

We think that while performing Lecompte's maneuver the anatomical substrate for SVC narrowing should also be addressed. First, the retro-aortic vein should be divided and brought anterior to aorta, to enable greater lateral mobilization of SVC. Second, the RPA reduction should be done to minimize the compression of SVC by it. Third, wedge resection of greater curvature of aorta, where feasible, can decrease the anterior displacement of right pulmonary artery. Fourth, low cannulation of SVC (below innominate junction) cans possible lead to presence of intact endothelium at site of narrowing, thereby leading to decrease propensity for thrombus formation.

\section{References}

1. Talwar S, Muthukkumaran S, Choudhary SK, Airan B (2014) The expanding indications for the Lecompte maneuver. World J Pediatr Congenit Heart Surg 5: 291-296. 